\title{
Reducing the risk of athlete burnout: Psychosocial, sociocultural, and individual considerations for coaches
}

\author{
Louise Davis', Andreas Stenling ${ }^{1,2,3}$, Henrik Gustafsson ${ }^{4}$, \\ Ralph Appleby ${ }^{5}$ and Paul Davis ${ }^{1}$
}

\begin{abstract}
Past research suggests that athletes' relationships with their coach can act as a risk factor in the development of burnout. Coaching practice may be enhanced through understanding the multidimensional factors that can augment the associations between coach-athlete relationship quality and athlete burnout. The present study explored both individual difference characteristics (gender, age, and sport level) and sociocultural factors (sport type) as moderators of this relationship. Our findings show statistically significant interaction effects for gender and age. Coaches and practitioners working with younger athletes and male performers in particular, are advised to work with strategies aiming to build relationships and reduce the risk of burnout.
\end{abstract}

\section{Keywords}

Age, coach-athlete relationships, gender

"I'm exhausted, I just can't wait to see my coach." -Ellie Simmons immediately after winning Paralympic $100 \mathrm{~m}$ freestyle gold (2012)

Post-race interviews are often venues for heartfelt expressions of gratitude from athletes acknowledging the role of significant people in their lives (e.g. parents, coaches). These sincere declarations highlight the pivotal position of coaches in supporting athletes' performance and development, as well as illustrate the theoretical postulates of the coach-athlete relationship being a central feature of an athlete's sport experience. ${ }^{1}$

Over the past 15 years, the content, quality, and functions of the coach-athlete relationship have been studied; in particular, four key relational constructs have been advanced in defining the quality of the coach-athlete relationship. ${ }^{2}$ Specifically, the coach-athlete relationship is considered a situational context characterised by a coach's and athlete's feelings of closeness (i.e. an emotional connection reflected in trust, like, respect), thoughts of commitment (i.e. motivation to maintain a close relationship over time), and complementarity behaviors (i.e. behaviors reflected in interactions that are responsive, relaxed, and friendly). In addition co-orientation characterises a common ground regarding coaches' and athletes' feelings, thoughts, and behaviours. These four relational constructs have been operationalised within the $3+1 \mathrm{Cs}$ model and shape the quality of the coach-athlete relationship. ${ }^{1,2}$

According to Jowett and Poczwardowski's ${ }^{3}$ integrated research model, the properties of the $3+1 \mathrm{Cs}$ model are said to influence and be influenced by a number of factors. For example, previous research has investigated both positive and negative consequent variables that are influenced by the quality of the

Reviewer: Svenja Wachsmuth (University of Tuebingen, Germany)

'Department of Psychology, Umeå University, Umeå, Sweden

${ }^{2}$ Department of Psychology, University of Gothenburg, Gothenburg, Sweden

${ }^{3}$ Department of Psychology, University of Otago, Dunedin, New Zealand ${ }^{4}$ Faculty of Arts and Social Sciences, Karlstad University, Sweden \& Norwegian School of Sport Sciences, Norway

${ }^{5}$ Department of Sport, Exercise, and Rehabilitation, Northumbria University, Newcastle upon Tyne, UK

\section{Corresponding author:}

Louise Davis, Department of Psychology, Umeå University, Umeå, Sweden.

Email: louise.davis@umu.se 
coach-athlete relationship. Specifically, studies have examined positive outcomes including sport and relationship satisfaction, ${ }^{4,5}$ motivation, ${ }^{6}$ well-being, ${ }^{7}$ and physical and cognitive performance. ${ }^{8}$ Recent research has also studied negative consequences including interpersonal conflict ${ }^{9}$ and athlete burnout. ${ }^{10}$

Athlete burnout has been defined as a multidimensional psychophysiological syndrome characterised by symptoms of emotional and physical exhaustion (i.e. perceived depletion of emotional and physical resources resulting from training and/or competition), reduced sense of sporting accomplishment (i.e. negative evaluation of ones sporting abilities and achievements), and the sport devaluation (i.e. a cynical attitude towards sport participation). ${ }^{11,12}$ Although this multidimensional definition is the most commonly used in sport psychology research, many researchers consider exhaustion to be the core of burnout. ${ }^{8,13}$ Numerous years of research have illustrated how burnout is associated with negative cognitive, motivational, and behavioural outcomes for athletes. ${ }^{14}$ For example, burnout has been associated with psychological stress, ${ }^{15}$ reduced coping skills and optimism ${ }^{16}$ as well as sport dropout. ${ }^{10}$

Past research suggests that athletes' negative interactions with their coach can act as a risk factor in the development of burnout ${ }^{10,17}$ and underlie poor coachathlete relationship quality. ${ }^{8,10,18}$ Specifically, Davis et al. ${ }^{8}$ and Isoard-Gautheur et al. ${ }^{10}$ found a negative link between coach-athlete relationship quality (i.e. captured through the interpersonal constructs of the 3Cs) and athlete burnout (i.e. captured through reduced sense of accomplishment, sport devaluation, and emotional and physical exhaustion). These findings highlight that athletes perceiving high quality coachathlete relationships reported lower levels of burnout in that they felt higher personal accomplishment, less negative feelings towards their sport, and lower emotional and physical exhaustion than those indicating poor quality relationships with their coach. In particular, both studies note relationship quality as a stronger predictor of the cognitive and emotional elements of athlete burnout.

Whilst these findings support the proposed theoretical associations between coach-athlete relationship quality and athlete burnout, coaching practice can be enhanced through increased understanding of the multidimensional factors that may augment the associations between the quality of the coach-athlete relationship and athlete burnout. ${ }^{3}$ In particular, bespoke interventions could be delivered if applied sport psychologists and coaches were better informed about individual difference characteristics (e.g. age, gender, and sport level) and sociocultural factors (e.g. type of sport) that influence the associations between relationship quality and burnout in sport. Enhanced applied practice may subsequently reduce the incidence of athlete burnout and minimise the effects of negative consequences as a result (e.g. sport dropout). Therefore, the current study was designed to explore both individual difference characteristics (i.e. age, gender, and sport level) and sociocultural factors (i.e. sport type) as moderators of the relationship between coach-athlete relationship quality and athlete burnout.

\section{Method}

\section{Participants and procedure}

A total of 210 (28.1\% females; $71.9 \%$ males) UK-based competitive athletes participated in the current study. The age of the athletes ranged from 15 to 31 years $(M=18 ; S D=2.3$, one athlete did not report age), they practiced on average $11.8 \mathrm{~h}(S D=4.9)$ per week, and had an average of 9.5 years $(S D=3.7)$ of competitive experience in their sport. The athletes were engaged in a variety of individual $(26 \%$; athletics, gymnastics, orienteering) and team (74\%; field hockey, football, handball, netball, rugby, volleyball) sports and their competitive level ranged from regional $(42.3 \%)$, national $(29.0 \%)$, to international $(28.1 \%)$. One athlete did not report competitive level.

Head coaches were contacted via email or by telephone, where the overall purpose of the study was explained and permission to administer a multi-section questionnaire to their athletes was sought. Information sheets outlining the aims of the study were provided to coaches and athletes prior to athletes granting written consent. For those athletes under the age of 18, parental information sheets were provided and parental consent was sought in addition to athletes providing their consent. Arrangements were made for the athletes to complete the multi-section questionnaire prior to a training session and independently from their coach and peers. Institutional ethics approval was granted from the associated University ethics committee.

\section{Instruments}

Demographic and background inventory. Participants provided a variety of demographic information including: age, gender, years of competitive experience, years played with current team, and level of sport competition.

Coach-athlete relationship quality. The 11-item CoachAthlete Relationship Questionnaire $\left(\mathrm{CART}-\mathrm{Q}^{19}\right)$ was employed to measure athletes' direct perception of the quality of their coach-athlete relationship. ${ }^{2}$ The direct perspective of the CART-Q has four items assessing closeness (e.g. "I like my coach"), three items assessing 
commitment (e.g. "I am committed to my coach") and four items assessing complementarity (e.g. "When I am coached by my coach, I am ready to do my best"). All CART-Q items were measured on a scale ranging from 1 ("Strongly Disagree") to 7 ("Strongly Agree"). We combined the three factors into a single score representing an overall measure of the coach-athlete relationship quality. ${ }^{19}$

Athlete Burnout. The 15-item Athlete Burnout Questionnaire $\left(\mathrm{ABQ}^{12}\right)$ was used to investigate athletes' level of burnout. The ABQ contains three subscales, including five items that measure emotional/physical exhaustion (e.g. "I feel overly tired from my sport participation"), five items that measure reduced sense of sport accomplishment (e.g. "I am not performing up to my ability in sport"), and five items that measure sport devaluation (e.g. "I don't care as much about my sport performance as I used to"). The stem for each item was "How often do you feel this way?" and participants responded on a scale ranging from 1 (almost never) to 5 (almost always).

\section{Statistical analysis}

Initial preliminary analysis were conducted whereby the data were screened for non-normality (i.e. skewness and kurtosis) and outliers (standardised $z$ scores $>3.29$, $p<.001$, Mahalanobis distances and the critical value of $\chi^{2}(4)=18.47 \quad(p<.001) .{ }^{20}$ Bivariate correlations were calculated to examine relationships among the variables. Omega coefficients $(\omega)$ were calculated to assess internal consistency of the scales. ${ }^{21}$

For the main analyses, we used IBM SPSS version 24 and the SPSS macro PROCESS ${ }^{22}$ to examine the direct and moderated associations between coachathlete relationship quality and athlete burnout. We present results from the unconditional and moderated regression analyses because the interpretation of direct effects change when an interaction term is included as a predictor in the model. In a simple moderation model (i.e. with one independent variable and one moderator), the direct effect of the independent variable on the dependent variable represents the association when the moderator is 0 . The direct effect of the moderator on the dependent variable represents the association when then independent variable is 0 . Hence, the direct effects in a moderated regression analysis are conditional effects. ${ }^{22}$

Continuous independent variables and moderators were mean centred prior to the moderated regression analyses to aid interpretation. ${ }^{23}$ Categorical moderators were competitive level (regional $=1$, national $=$ 2 , international $=3$ ), gender $($ female $=0$, male $=1$ ), and type of sport (individual $=0$, team sport $=1$ ). To examine the moderating effect of age, we used the Johnson-Neyman $(\mathrm{JN})$ technique. ${ }^{22}$ The $\mathrm{JN}$ technique identifies points along the age continuum where the association between coach-athlete relationship quality and burnout transitioned between not statistically significant and statistically significant. Hence, these points demarcate regions of significance of the effect of the predictor on the outcome. Separate analyses were conducted for each moderator. The significance level was set to 0.05 in the analyses.

\section{Results}

\section{Preliminary analysis}

Initial analyses indicated four univariate outliers with $z$ scores larger than 3.29 and two multivariate outliers with Mahalanobis distances larger than 18.47. We excluded these six outliers from further analysis. Two additional athletes were excluded from the analyses because they did not have data on the moderators. As such, the sample size in moderated regression analyses was 202 athletes. All variables showed low skewness and kurtosis $(< \pm 1)$ and internal consistency (omega coefficient) ranged from 0.73 to 0.92 . Descriptive statistics and correlations for the whole sample are displayed in Table 1 (descriptive statistics for the subgroups are displayed in Table 2). Coach-athlete relationship quality was negatively associated with all

Table I. Descriptive statistics, correlations, and internal consistency (omega coefficients $[\omega]$ ).

\begin{tabular}{lllllllrr}
\hline & RA & EX & SD & CAR & $M$ & SD & Skew & Kurt \\
\hline RA & 0.73 & & & & 2.31 & 0.63 & 0.042 & -0.320 \\
EX & $0.154^{*}$ & 0.88 & & & 2.41 & 0.75 & 0.349 & -0.294 \\
SD & $0.430^{*}$ & $0.444^{*}$ & 0.79 & & 1.72 & 0.64 & 0.733 & -0.181 \\
CAR & $-0.26 I^{*}$ & $-0.357^{*}$ & $-0.252^{*}$ & 0.92 & 5.44 & 0.97 & -0.663 & 0.082 \\
\hline
\end{tabular}

Note: $N=202$. Omega coefficient $(\omega)$ displayed in the diagonal. RA: reduced sense of sporting accomplishment, EX: emotional and physical exhaustion, SD: sport devaluation; CAR: coach-athlete relationship. 
Table 2. Descriptive statistics for the subgroups used in the moderated regression analyses.

\begin{tabular}{|c|c|c|c|c|c|c|c|c|c|c|c|c|c|c|}
\hline & \multicolumn{6}{|c|}{ Competitive level } & \multicolumn{4}{|c|}{ Type of sport } & \multicolumn{4}{|l|}{ Sex } \\
\hline & \multicolumn{2}{|c|}{$\begin{array}{l}\text { Regional } \\
(n=86)\end{array}$} & \multicolumn{2}{|c|}{$\begin{array}{l}\text { National } \\
(n=59)\end{array}$} & \multicolumn{2}{|c|}{$\begin{array}{l}\text { International } \\
(n=57)\end{array}$} & \multicolumn{2}{|c|}{$\begin{array}{l}\text { Individual } \\
(n=54)\end{array}$} & \multicolumn{2}{|c|}{$\begin{array}{l}\text { Team } \\
(n=148)\end{array}$} & \multicolumn{2}{|c|}{$\begin{array}{l}\text { Females } \\
(n=58)\end{array}$} & \multicolumn{2}{|c|}{$\begin{array}{l}\text { Males } \\
(n=144)\end{array}$} \\
\hline & $M$ & $S D$ & $M$ & $S D$ & $M$ & $S D$ & $M$ & $S D$ & $M$ & $S D$ & $M$ & $S D$ & $M$ & $S D$ \\
\hline RA & 2.41 & 0.62 & 2.43 & 0.61 & 2.04 & 0.59 & 2.32 & 0.68 & 2.31 & 0.61 & 2.35 & 0.59 & 2.29 & 0.65 \\
\hline EX & 2.47 & 0.69 & 2.33 & 0.75 & 2.40 & 0.85 & 2.01 & 0.74 & 2.55 & 0.71 & 2.37 & 0.88 & 2.42 & 0.70 \\
\hline SD & 1.74 & 0.61 & 1.79 & 0.60 & 1.62 & 0.73 & 1.62 & 0.57 & 1.75 & 0.67 & 1.60 & 0.54 & 1.77 & 0.68 \\
\hline CAR & 5.24 & 0.84 & 5.43 & 0.96 & 5.73 & 1.09 & 6.02 & 0.56 & 5.22 & 1.00 & 5.42 & 1.04 & 5.44 & 0.95 \\
\hline
\end{tabular}

RA: reduced sense of sporting accomplishment; EX: emotional and physical exhaustion; SD: sport devaluation; CAR: coach-athlete relationship.

three burnout dimensions, whereas the three burnout dimensions were all positively correlated.

\section{Main analyses}

Results from the unconditional regression analyses are displayed in Table 3 and the moderated regression analyses are displayed in Table 4. A higher quality coachathlete relationship was associated with lower scores on all three burnout dimensions.

Competitive level. Athletes competing at the international level reported lower levels of reduced sense of accomplishment compared to athletes competing at lower levels $(B=-0.30, p<0.001)$. No statistically significant interaction effect of the coach-athlete relationship and competitive level were found.

Type of sport. Team sport athletes reported higher levels of exhaustion than individual sport athletes $(B=0.37$, $p=0.002$ ), but there was no statistically significant interaction effect between the coach-athlete relationship and type of sport.

Gender. There was a statistically significant interaction effect of the coach-athlete relationship and gender on reduced sense of sporting accomplishment $(B=-0.32$, $p=0.003$ ) indicating that the association was stronger for males compared to females (see Figure 1).

Age. There was a positive and statistically significant interaction effect of the coach-athlete relationship and age on reduced sense of sporting accomplishment $(B=0.04, p=0.049)$. The association between the coach-athlete relationship and reduced sense of sporting accomplishment was statistically significant for athletes aged 19 years and younger, but not for athletes older than 19 years (see Figure 2).

\section{Discussion}

In attempting to decrease the incidence of athlete burnout, coaching and applied practice would benefit from increased understanding of the nuances that influence an athlete's susceptibility. The present study explored individual difference characteristics (i.e. age, gender, and sport level) and sociocultural factors (i.e. sport type) as moderators of the relationship between coach-athlete relationship quality and athlete burnout symptoms. In line with earlier research, ${ }^{8,10,18}$ higher quality coach-athlete relationships were associated with reports of fewer symptoms of burnout. These findings underline the importance of psychosocial factors associated with the risk of burnout in competitive athletes, as there were no interaction effects found for competition levels and type of sport. Therefore, optimising coach-athlete relationship quality is applicable in attempts to minimise the risk of burnout across various types and levels of sport competition.

Previous research posits that individual athletes are at greater risk of burnout ${ }^{24,25}$ as demands and pressures on the individual can be greater than in teams where the load may be shared, and teammates can act as a buffer by providing social support. ${ }^{26}$ Contrary to this position, Gustafsson and colleagues ${ }^{27}$ found a higher prevalence of burnout symptoms in team sports than in individual sports. The present study's findings also note that team sport athletes score higher on the core aspect of the burnout construct of exhaustion. ${ }^{14}$ This underlines the importance of social relations within athlete burnout. ${ }^{28}$

Across numerous studies, athletes' relationships with their teammates and coaches emerge as a key factor in the development of burnout. ${ }^{29}$ In particular, anti-social behavior within teams has been observed to predict burnout, whilst pro-social behavior negatively predicts burnout. ${ }^{26}$ Smith et al. ${ }^{28}$ found that intra-team conflict significantly correlates with burnout in adolescent athletes. Conflict is associated with lower levels of relationship quality and team sport athletes report less 


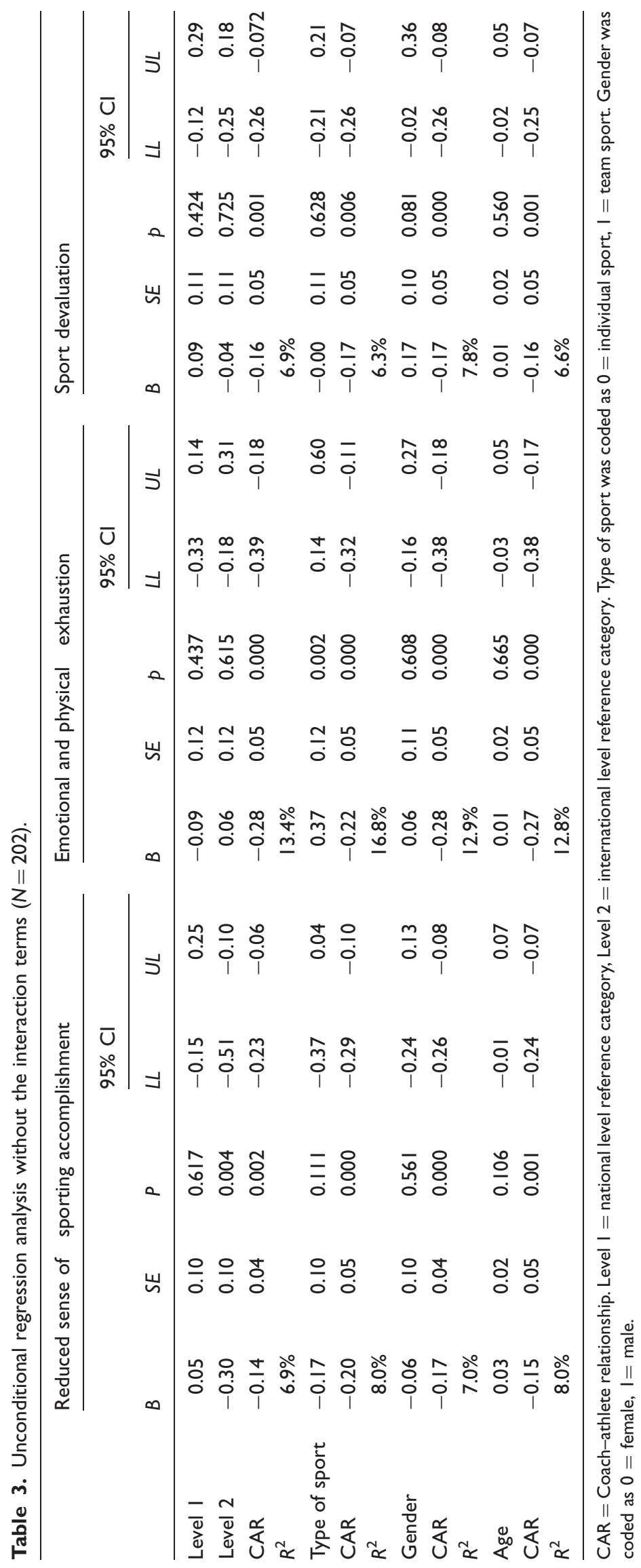




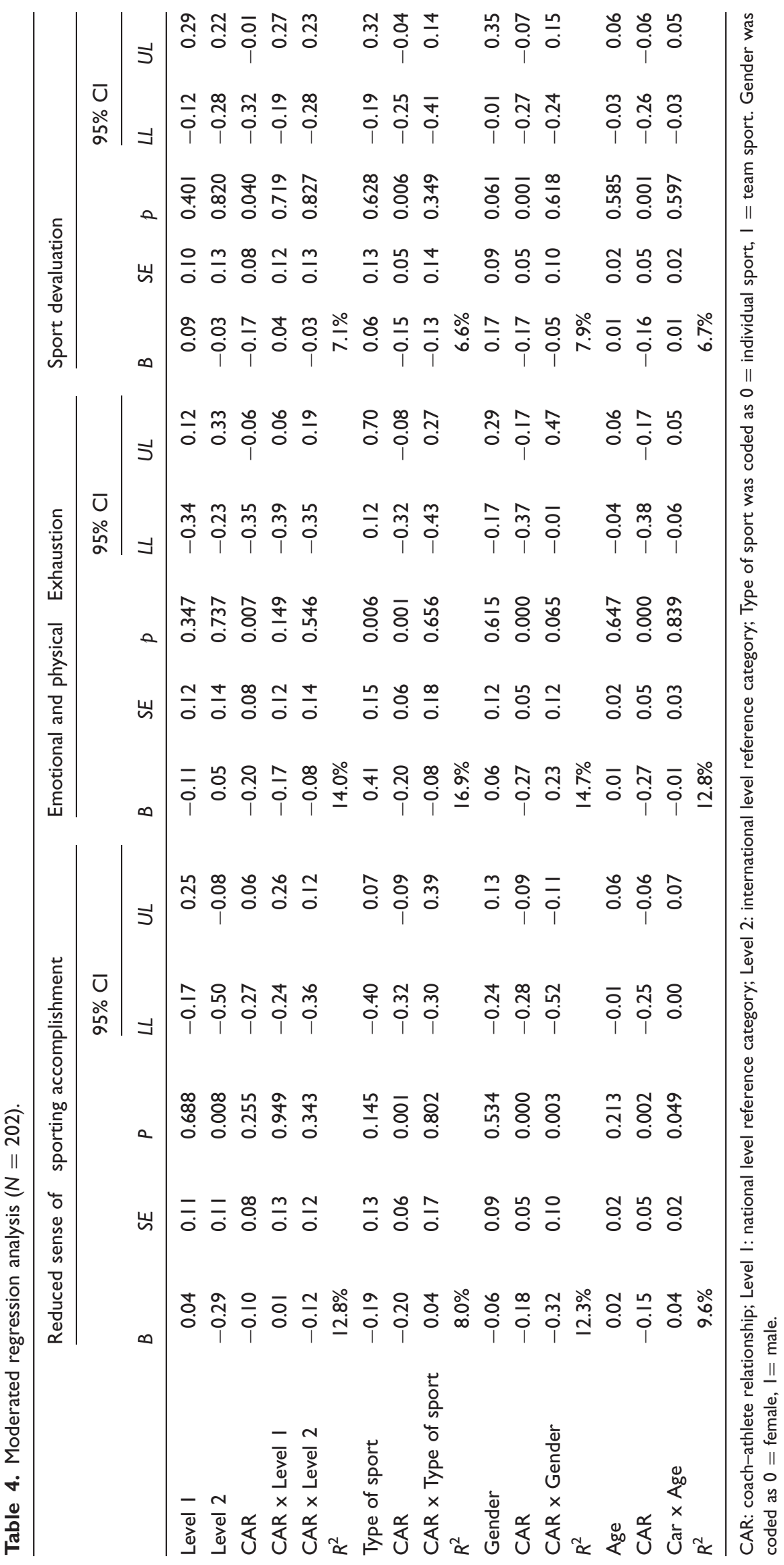




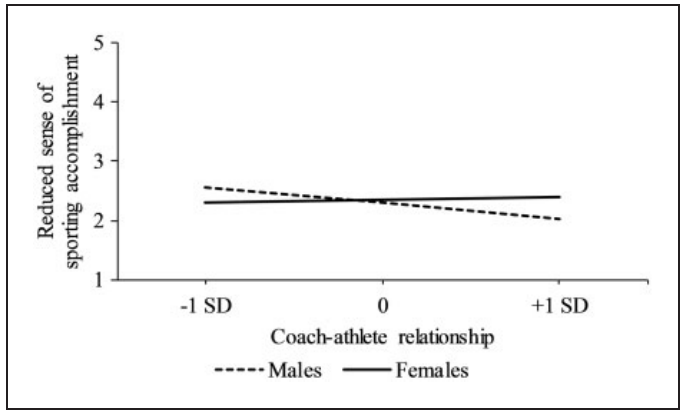

Figure I. Moderating effect of gender in the association between the coach-athlete relationship and reduced sense of sporting accomplishment.

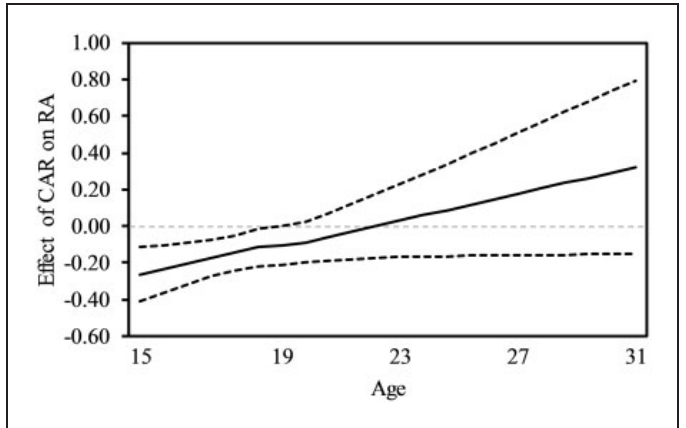

Figure 2. Moderating effect of age in the association between the coach-athlete relationship and reduced sense of sporting accomplishment. The $\mathrm{Y}$-axis shows unstandardised regression coefficients and the $X$-axis shows the age range in the sample. The black line represents the association between the coachathletes relationship and reduced sense of sporting accomplishments at different ages. The black dashed lines represent the upper and lower limits of the $95 \%$ confidence interval.

closeness, commitment, and complementarity with their coaches than athletes from individual sports. ${ }^{30}$ If coaches fail to establish good quality relationships with each athlete comprising their team, individual members may not commit to the team's shared vision and instead focus upon their own self-promoting agendas at the expense of team cohesion. ${ }^{31}$ Coaches can develop social and task cohesion within a team by developing perceptions of closeness through shared emotional experiences (e.g. performance success and failure) and the use of effective emotion regulation strategies with their athletes during intense competition and training. ${ }^{32}$

To enhance motivation and minimise conflict, coaches are encouraged to open channels of communication through the use of relationship enhancement strategies as outlined in the COMPASS model. ${ }^{33}$ Communication strategies enhance the quality of relationships as well as improve motivation and perceptions of support, both of which have been shown to relate with athlete burnout. ${ }^{18}$ Future intervention studies targeting the use of effective communication strategies would provide support for coach education programs that center on the development of relationship quality in optimising athletes' health and sport accomplishments.

The moderated regression analyses showed that the association between the coach-athlete relationship and reduced sense of sporting accomplishment was stronger for younger athletes. These findings are in support of previous research indicating that young athletes are often more prone to burnout. ${ }^{34}$ Further, athletes competing at the international level reported lower levels of reduced sense of accomplishment compared to athletes competing at lower levels. This potentially is a marker of characteristics developed from competitive success and accomplishments required for athletes to progress to international level competition; it may also indicate the maturity, accrued experience, and independence necessary for international level competition. Young athletes have been found to be more dependent upon their coaches and less involved in making decisions related to training load. ${ }^{35}$ As athletes mature, relationships evolve; high quality relationships are typified by mutual respect and offer greater collaboration in determining training intensity. To promote athlete wellbeing, coaches can develop interpersonal environments that encourage athletes at an earlier age to express their thoughts and feelings associated with training, beyond the technical and/or tactical aspects. Open communication about broader aspects of training can maximise performance and optimise the influence of antecedents associated with athlete burnout (e.g. motivation).

The moderated regression analyses also showed that the association between the coach-athlete relationship and reduced sense of sporting accomplishment was stronger for male athletes. That said, research examining the exhaustion dimension of athlete burnout have found that females often report greater symptoms than males. ${ }^{27}$ Research focusing upon demographic factors in athlete burnout is scarce and the role of reduced sense of sporting accomplishment is less established than the dimensions of exhaustion and cynicism (i.e. sport devaluation ${ }^{12}$ ). Future research should consider evaluating the role of gender further.

Future research may also seek to explore the influence of discrepancies between coaches' and athletes' perspectives of associations linking coach-athlete relationship quality and burnout across age levels and types of sports. The cross-sectional research design and the use of unidirectional measurements are limitations of the present study; increased understanding of the interpersonal nature of the coach-athlete relationship would benefit from bi-directional measurement and longitudinal research. 
In summary, the findings of the present study highlight that coaches may play a central role in athletes' susceptibility to burnout. Coaches and applied sport psychologists working with younger athletes are advised that these athletes are at greater risk of developing burnout. In particular, male athletes can benefit from good quality coach-athlete relationships in preventing a reduced sense of accomplishment. Building and maintaining high quality relationships may optimise the health and high performance of athletes.

\section{Declaration of conflicting interests}

The author(s) declared no potential conflicts of interest with respect to the research, authorship, and/or publication of this article.

\section{Funding}

The author(s) received no financial support for the research, authorship, and/or publication of this article.

\section{References}

1. Jowett S. Coaching effectiveness: the coach-athlete relationship at its heart. Cur Opin Psychol 2017; 16: 154-158.

2. Jowett $\mathrm{S}$. Interdependence analysis and the $3+1 \mathrm{Cs}$ in the coach- athlete relationship. In: Jowett S and Lavallee D (eds) Social psychology in sport. Champaign, IL: Human Kinetics, 2007, pp.63-77.

3. Jowett $\mathrm{S}$ and Poczwardowski A. Understanding the coach-athlete relationship. In: Jowett $\mathrm{S}$ and Lavallee D (eds) Social psychology in sport. Champaign, IL: Human Kinetics, 2007, pp.3-14.

4. Davis L, Jowett $\mathrm{S}$ and Lafrenière MAK. An attachment theory perspective in the examination of relational processes associated with coach-athlete dyads. J Sport Exerc Psychol 2013; 35: 156-167.

5. Lorimer R. Coaches' satisfaction with their athletic partnerships. Int J Coaching Sci 2009; 3: 55-64.

6. Adie JW and Jowett S. Meta-perceptions of the coachathlete relationship, achievement goals, and intrinsic motivation among sport participants. J Appl Social Psychol 2010; 40: 2750-2773.

7. Felton L and Jowett S. Attachment and well-being: the mediating effects of psychological needs satisfaction within the coach-athlete and parent-athlete relational contexts. Psychol Sport Exerc 2013; 14: 57-65.

8. Davis L, Appleby R, Davis PA, et al. The role of coachathlete relationship quality in team sport athletes' psychophysiological exhaustion: implications for physical and cognitive performance. J Sports Sci 2018; 36: 19851982.

9. Wachsmuth S, Jowett S and Harwood CG. On understanding the nature of interpersonal conflict between coaches and athletes. J Sports Sci 2018; 36: 1-8.

10. Isoard-Gautheur S, Trouilloud D, Gustafsson H, et al. Associations between the perceived quality of the coachathlete relationship and athlete burnout: An examination of the mediating role of achievement goals. Psychol Sport Exerc 2016; 22: 210-217.

11. Raedeke TD. Is athlete burnout more than just stress? A sport commitment perspective. J Sport Exerc Psychol 1997; 19: 396-417.

12. Raedeke TD and Smith AL. Development and preliminary validation of an athlete burnout measure. J Sport Exerc Psychol 2001; 23: 281-306.

13. Lundkvist E, Gustafsson H, Davis PA, et al. The temporal relations across burnout dimensions in athletes. Scan J Med Sci Sports 2018; 28: 1215-26.

14. Gustafsson H, DeFreese JD and Madigan DJ. Athlete burnout: Review and recommendations. Cur Opin Psychol 2017; 16: 109-113.

15. Gustafsson H, Davis P, Skoog T, et al. Mindfulness and its relationship with perceived stress, affect, and burnout in elite junior athletes. J Clin Sport Psychol 2015; 9: 263-281.

16. Gustafsson $\mathrm{H}$ and Skoog $\mathrm{T}$. The mediational role of stress in the relation between optimism and burnout in competitive athletes. Anx Stress Cop 2012; 25: 183-200.

17. DeFreese J and Smith AL. Athlete social support, negative social interactions, and psychological health across a competitive sport season. J Sport Exerc Psychol 2014; 36: 619-630.

18. Gustafsson H, Hassmén P, Kenttä G, et al. A qualitative analysis of burnout in elite Swedish athletes. Psychol Sport Exerc 2008; 9: 800-816.

19. Jowett $\mathrm{S}$ and Ntoumanis $\mathrm{N}$. The coach-Athlete relationship questionnaire (CARTQ): Development and initial validation. Scan J Med Sci Sports 2004; 14: 245-257.

20. Tabachnick BG and Fidell LS. Using multivariate statistics, 5th ed. New York, NY: Allyn and Bacon, 2007.

21. McDonald R. Test theory: a unified treatment. Mahwah, NJ: Lawrence Erlbaum Associates, 1999.

22. Hayes AF. Introduction to mediation, moderation, and conditional process analysis: a regression-based approach. New York, NY: The Guilford Press, 2013.

23. Aiken LS, West SG and Reno RR. Multiple regression: testing and interpreting interactions. New York, NY: Sage, 1991.

24. Coakley J. Burnout among adolescent athletes: a personal failure or social problem? Soc Sport J 1992; 9: 271-285.

25. Smith RE. Toward a cognitive-affective model of athletic burnout. J Sport Psychol 1986; 8: 36-50.

26. Al-Yaaribi A and Kavussanu M. Teammate prosocial and antisocial behaviors predict task cohesion and burnout: the mediating role of affect. J Sport Exerc Psychol 2017; 39: 199-208.

27. Gustafsson H, Kenttä G, Hassmén P, et al. Prevalence of burnout in competitive adolescent athletes. Sport Psychol 2007; 21: 21-37.

28. Smith AL, Gustafsson H and Hassmén P. Peer motivational climate and burnout perceptions of adolescent athletes. Psychol Sport Exerc 2010; 11: 453-460.

29. Appleby R, Davis PA, Davis L, et al. Examining perceptions of teammates' burnout and training hours in athlete burnout. J Clin Sport Psychol 2018; 12: 1-30. 
30. Rhind DJ, Jowett S and Yang SX. A comparison of athletes' perceptions of the coach-athlete relationship in team and individual sports. $J$ Sport Beh 2012; 35: 433-452.

31. Price MS and Weiss MR. Relationships among coach leadership, peer leadership, and adolescent athletes' psychosocial and team outcomes: a test of transformational leadership theory. $J$ Appl Sport Psychol 2013; 25: 265-279.

32. Davis PA and Davis L. Emotions and emotion regulation in coaching. In: Davis P (ed.) The psychology of effective coaching and management. New York, NY: Nova Science Publishers Inc., 2016, pp.285-306.

33. Rhind DJ and Jowett S. Linking maintenance strategies to the quality of coach-athlete relationships. Int J Sport Psychol 2011; 42: 55-68.

34. Maslach C, Schaufeli WB and Leiter MP. Job burnout. Ann Rev Psychol 2001; 52: 397-422.

35. Lorimer R and Jowett S. Coaches. Routledge companion to sport and exercise psychology. New York, NY: Routledge, 2014, pp.171-186. 